\title{
Stereotactic body radiotherapy for early stage non-small cell lung cancer in patients with subclinical interstitial lung disease
}

\author{
Yuanjun Liu ${ }^{1,2 \#}$, Yaoyao $\mathrm{Zhu}^{2 \#}$, Ran $\mathrm{Wu}^{2 \#}$, Min $\mathrm{Hu}^{2}$, Lingnan Zhang ${ }^{3}$, Qingren $\mathrm{Lin}^{4}$, Denghu Weng ${ }^{4}$, \\ Xiaojiang Sun ${ }^{4}$, Yu Liu ${ }^{2}$, Yaping $\mathrm{Xu}^{1,2,4}$ \\ ${ }^{1}$ First Clinical Medical School, Wenzhou Medical University, Wenzhou, China; ${ }^{2}$ Department of Radiation Oncology, Shanghai Pulmonary Hospital, \\ Tongji University School of Medicine, Shanghai, China; ${ }^{3}$ Department of Radiology, Cancer Hospital of University of Chinese Academy of Sciences \\ (Zhejiang Cancer Hospital), Institute of Cancer and Basic Medicine (IBMC), Chinese Academy of Sciences, Hangzhou, China; ${ }^{4}$ Department of \\ Radiation Oncology, Cancer Hospital of University of Chinese Academy of Sciences (Zhejiang Cancer Hospital), Institute of Cancer and Basic \\ Medicine (IBMC), Chinese Academy of Sciences, Hangzhou, China \\ Contributions: (I) Conception and design: Y Xu, Y Liu; (II) Administrative support: Y Xu; (III) Provision of study materials or patients: X Sun, Q \\ Lin, Y Liu; (IV) Collection and assembly of data: Y Zhu, R Wu, L Zhang, D Weng; (V) Data analysis and interpretation: Y Liu, Y Zhu, M Hu; (VI) \\ Manuscript writing: All authors; (VII) Final approval of manuscript: All authors. \\ "These authors contributed equally to this work. \\ Correspondence to: Yaping Xu. First Clinical Medical School, Wenzhou Medical University, University Town, Chashan, Wenzhou, China; Department \\ of Radiation Oncology, Shanghai Pulmonary Hospital, Tongji University School of Medicine, No. 507 Zhengmin Road, Shanghai, China. \\ Email: xuyaping1207@163.com; Yu Liu. Department of Radiation Oncology, Shanghai Pulmonary Hospital, Tongji University School of Medicine; \\ No. 507 Zhengmin Road, Shanghai, China. Email: johnyuliu@sina.com.
}

Background: For lung cancer patients with subclinical (untreated and asymptomatic) interstitial lung disease (ILD), there is a lack of relatively safe and effective treatment. Stereotactic body radiation therapy (SBRT) can achieve a high level of tumor control with low toxicity in early-stage non-small cell lung cancer (NSCLC). This study aimed to evaluate the efficacy and toxicity of early stage NSCLC patients with subclinical ILD receiving SBRT.

Methods: A total of 109 early stage NSCLC patients receiving SBRT treatment between December 2011 and August 2016 were reviewed in our institutions; patients with clinical ILD were excluded. The median dose of SBRT was 50 Gy in 5 fractions. The median biologically effective dose (BED; $\alpha / \beta=10)$ was 100 Gy (range, 72-119 Gy). An experienced radiation oncologist and an experienced radiologist reviewed the presence of subclinical ILD in the CT findings before SBRT. The relationships among the efficacy, radiation-induced lung injury (RILI) and subclinical ILD were explored.

Results: In all, 38 (34.9\%) of 109 patients were recognized with subclinical ILD before SBRT, 48 (44.0\%) of 109 patients were recognized with grade 2-5 RILI after SBRT, and 18 (47.4\%) of 38 patients with subclinical ILD were observed with grade 2-5 RILI. Subclinical ILD was not a significant factor of grade 2-5 RILI ( $\mathrm{P}=0.608$ ); however, 3 patients had extensive RILI, and they all suffered from subclinical ILD. Dosimetric factor of the lungs, such as mean lung dose (MLD) was significantly related with Grade 2-5 RILI in patients with subclinical ILD $(\mathrm{P}=0.042)$. The progression-free survival (PFS) rates at 3 years in the subclinical ILD patients and those without ILD were $61.6 \%$ and $66.8 \%$, respectively $(\mathrm{P}=0.266)$.

Conclusions: Subclinical ILD was not a significant factor for RILI or PFS in early stage NSCLC patients receiving SBRT; however, patients with subclinical ILD receiving SBRT may experience uncommon extensive RILI.

Keywords: Non-small cell lung cancer (NSCLC); outcome; stereotactic body radiotherapy (SBRT); toxicity; subclinical interstitial lung disease

Submitted Aug 12, 2020. Accepted for publication Nov 29, 2020.

doi: $10.21037 /$ tlcr-20-1050

View this article at: http://dx.doi.org/10.21037/tlcr-20-1050 


\section{Introduction}

Interstitial lung disease (ILD) is a group of acute, noninfectious, and chronic diffuse parenchymal lung disorders, and can be subdivided into two groups, idiopathic pulmonary fibrosis (IPF), and ILD other than IPF (other ILD). ILD is considered to be associated with more than 150 clinical factors and/or conditions (1). Although it includes a group of heterogeneous diseases, it is always associated with restrictive defects in pulmonary function testing and reduction of gas exchange. It is interesting to note that one of the independent risk factors for the development of lung cancer is the diagnosis of ILD (2). However, regardless of the treatment-related toxic side effects or acutely worsened ILD patients, lung cancer complicated with ILD is a highrisk group for treatment (3-6). These adverse effects can be severe, and even fatal in extreme cases. Even for early stage primary lung cancer patients, interstitial pneumonia cause about half of the postoperative 30-day deaths (7). Meanwhile, in the patients with co-existing ILD, the frequency of acute exacerbation after conventional radiotherapy has been reported to be around $25 \%$ (8).

As one of the common radical treatment methods for non-small cell lung cancer (NSCLC), stereotactic body radiotherapy (SBRT) has the characteristics of convenience, strong tolerance, and high efficacy. In recent years, SBRT has rapidly replaced conventional radiotherapy as an effective treatment for early NSCLC (9). It has been an attractive option in patients with significant medical co-morbidities for its high local control rate and non-invasive features. However, few reports mention the treatment outcomes of SBRT in early stage NSCLC patients with ILD.

To our knowledge, this is the first study to explore the relationship between ILD and the outcome in early stage NSCLC with subclinical (untreated and asymptomatic) ILD treated with SBRT. The purpose of this study was to evaluate the efficacy and toxicity of SBRT in the early stage NSCLC patients with co-existing subclinical ILD, and to explore whether subclinical ILD is a predict factor for radiation-induced lung injury (RILI).

We present the following article in accordance with the STROBE reporting checklist (available at http://dx.doi. org/10.21037/tlcr-20-1050).

\section{Methods}

\section{Patients}

A retrospective analysis was performed in 109 consecutive patients with histologically proven stage I NSCLC treated with SBRT in Shanghai Pulmonary Hospital and Cancer Hospital of University of Chinese Academy of Sciences (Zhejiang Cancer Hospital) between December 2011 and August 2016. Patients with subclinical ILD, which was defined as having asymptomatic ILD on computed tomography (CT) and no need for treatment, were included for SBRT. However, patients with clinical ILD, which was defined as having clinical symptoms or requiring treatment, were excluded. All patients were evaluated by experienced thoracic cancer surgeons to determine medically inoperable patients. This retrospective study excluded patients whose tumors were previously subjected to radiotherapy. All patients were confirmed primary NSCLC by biopsy or clinical diagnosis according to medical history and imaging features. There were no restrictions on lung function tests.

\section{Grade according to the severity of subclinical ILD}

According to a previous study (10), subclinical ILD were graded based on standard CT findings and patients were divided into four groups: no evidence of ILD (score 0), slight ILD (score 1), mild ILD (score 2), and moderate ILD (score 3). An experienced radiation oncologist and an experienced radiologist reviewed all CT images and CT findings were scored. Slight ILD was defined as focal or unilateral ground-glass attenuation, focal or unilateral reticulation, and patchy ground glass abnormalities (less than $5 \%$ of the lungs). Mild ILD was defined as nondependent ground-glass abnormalities affecting more than $5 \%$ of any lung zone, nondependent reticular abnormalities, diffuse centrilobular nodularity with ground glass abnormalities, honeycombing, traction bronchiectasis, non-emphysematous cysts, and architectural distortion. Moderate ILD was defined as bilateral fibrosis in multiple lobes associated with honeycombing and traction bronchiectasis in a subpleural distribution. Pulmonary infection was evaluated by blood tests, sputum cultures or bronchoalveolar lavage (BAL), and eliminated through antibiotic response. The presence of chronic obstructive pulmonary disease (COPD) and pulmonary emphysema $(\mathrm{PE})$ were also reviewed.

\section{Grade according to the severity of radiation-induced lung injury}

Retrospective assessment of patient toxicity was performed according to the National Cancer Institute Common 
Toxicity Criteria for Adverse Events version 5 (CTCAE v5.0) (11), which consists of five grades: Grade 1 mild: asymptomatic; clinical or diagnostic observations only; intervention not indicated; Grade 2 moderate: symptomatic; medical intervention indicated; limiting instrumental activities of daily living (ADL) (instrumental ADL refers to shopping for groceries or clothes, cooking, financial management, operating mobile phones, etc.); Grade 3: severe symptoms; limiting selfcare ADL (selfcare ADL refers to bathing, dressing and undressing, feeding self, using the toilet, taking medications, and not being bedridden); oxygen indicated; Grade 4: life-threatening respiratory compromise and indicated for urgent intervention; Grade 5: death. Acute radiological changes in lung include patchy ground glass opacities (GGO), diffuse GGO, patchy consolidation, and diffuse consolidation; late radiological changes include a modified conventional pattern (defined as traction bronchiectasis, consolidation and volume loss), mass-like fibrosis, and scar-like fibrosis $(12,13)$.

\section{SBRT treatment}

All patients received simulation based on 4D-CT. A vacuum immobilization bag was used to immobilize the patient's body. At our centers, the gross tumor volume (GTV) of each patient was drawn as a visible tumor on CT lung window imaging. Internal target volume (ITV) was outlined according to GTV and edited at the lung window with reference to all the expiratory and inspiratory phases of 4DCT in order to involve the full movement of the tumor. The planned target volume (PTV) was outlined based on ITV plus a $5-\mathrm{mm}$ setup margin. The dose prescription required that $99 \%$ of the ITV and $100 \%$ of the composite GTV should receive the prescribed dose, and that $95 \%$ of the PTV receive the prescribed dose. Organs at risk (OARs) include the trachea and proximal bronchial tree (TPBT), heart, great vessels, esophagus, both lungs, spinal cord, chest wall, and brachial plexus. Treatment was delivered using a 6- or 10-MV linear accelerator with either multiple static intensity-modulated radiation therapy or a $3 \mathrm{D}$ conformal treatment field. Cone beam CT was performed before each treatment fraction. Medical physicists formulated the treatment plans based on the prescribed dosage of the treating and chief physicians. Dose limits on critical organs were according to National Comprehensive Cancer Network (NCCN) guidelines. The median dose of SBRT was 50 Gy in 5 fractions. The median biologically effective dose (BED; $\alpha / \beta=10)$ was 100 Gy (range, $72-119$ Gy).

\section{Follow-up}

All patients were assessed every 3 months for the first 2 years after SBRT and every 6 months thereafter. Chest CT scans were performed at each follow-up to monitor tumor response and the development of RILI. The frequency of CT scan increased if there was suspicion of tumor recurrence, and the relapse was verified with ${ }^{18}$ F-FDG PET/CT or biopsy. The National Cancer Institute's CTCAE Version 5.0 was used to evaluate patient's toxicity.

\section{Statistical analysis}

Statistical analyses were performed using SPSS software (version 22.0, IBM SPSS, New York, USA). Significance was defined as a $P$ value less than 0.05 . The relationships between RILI and clinical factors were studied by the $\chi^{2}$ test and Fisher's exact probability test. The relationships between RILI and dosimetric factors were analyzed by two-sample $t$-test and Mann-Whitney $\mathrm{U}$ test. In terms of dosimetric factors, the total normal lung volume was defined as the total lung volume minus ITV. Dose parameters including the volumes of the lungs receiving more than a threshold dose and mean lung dose (MLD) were generated from a dose volume histogram (DVH) of the total normal lungs. The correlations between the occurrence of RILI and these factors were studied. The Kaplan-Meier method was used to calculate progression-free survival (PFS) and overall survival (OS) of patients from the first day of SBRT. Log-rank test was used to assess the statistical significance between the actuarial curves.

All procedures performed in this study involving human participants were in accordance with the Declaration of Helsinki (as revised in 2013). The study was approved by institutional/regional/national ethics/committee/ethics board of Shanghai Pulmonary Hospital (No.: L20-320Y) and individual consent for this retrospective analysis was waived.

\section{Results}

A total of 109 stage I NSCLC patients were enrolled in this study with a median follow-up of 55.1 months (range, 44.1-65.9 months). The patients' clinical characteristics are shown in Table 1. Eighty patients were male (73.4\%) while 29 were female (26.6\%). Patients were generally advanced in age, with a median age of 75 years at the start 
Table 1 Clinical characteristics of the 109 enrolled patients

\begin{tabular}{|c|c|}
\hline Characteristics & Patients \\
\hline Gender (M/F) & $80 / 29$ \\
\hline Age (years, median) & $47-88,75$ \\
\hline $\mathrm{PS}(0 / 1 / 2 / 3 / 4)$ & $24 / 81 / 4 / 0 / 0$ \\
\hline Subclinical ILD (no/yes) & $71 / 38$ \\
\hline COPD (no/yes) & $84 / 25$ \\
\hline PE (no/yes) & $84 / 25$ \\
\hline \multicolumn{2}{|l|}{ Tumor location } \\
\hline Left upper lobe & 32 \\
\hline Left lower lobe & 15 \\
\hline Right upper lobe & 41 \\
\hline Right middle lobe & 4 \\
\hline Right lower lobe & 17 \\
\hline \multicolumn{2}{|l|}{ Tumor size (cm) } \\
\hline$\leq 3$ & 89 \\
\hline$>3$ & 20 \\
\hline \multicolumn{2}{|c|}{ Histology of primary lung cancers } \\
\hline Squamous cell carcinoma & 27 \\
\hline Adenocarcinoma & 33 \\
\hline Large cell carcinoma & 0 \\
\hline Unclassified NSCLC & 16 \\
\hline Clinically diagnosed & 33 \\
\hline
\end{tabular}

PS, performance status; ILD, interstitial lung disease; COPD, chronic obstructive pulmonary disease; PE, pulmonary emphysema; NSCLC, non-small cell lung cancer. of treatment.

The frequency of grade 0-5 RILI was summarized in Table 2. Thirty-eight (35\%) of 109 patients was recognized with subclinical ILD on the pre-SBRT CT findings: moderate ILD (score 3 ) in 4 patients, mild ILD (score 2) in 6 patients had, slight ILD (score 1) in 28 patients, and no evidence of ILD (score 0) in 71 patients. Furthermore, $48(44.0 \%)$ of 109 patients had Grade 2 or higher RILI: 42 patients were Grade 2, 3 patients were Grade 3, 2 patients were Grade 4, and 1 patient was Grade 5. Symptomatic Grade 2 or higher RILI appeared at 4 months (range, 1.012.0 months) after SBRT. Grade 2 or higher RILI appeared in $18(47.4 \%)$ of 38 patients with subclinical ILD, 10 (40.0\%) of 25 patients with COPD, and 12 (48.0\%) of 25 patients with PE. Subclinical ILD was not a significant factor for the occurrence of Grade 2 or higher RILI, and the rates of COPD and PE were also not significant.

Three patients appeared to have extensive pneumonia beyond the irradiation area involving the contralateral lung, and they all had subclinical ILD on CT before SBRT. The characteristics of these patients were shown in Table 3. The incidence rate of extensive RILI in patients with subclinical ILD was higher than that without $(\mathrm{P}=0.040)$. Two patients were Grade 4 RILI: one patient died due to possible treatment-related pulmonary infection and respiratory failure, although it was difficult to confirm if this patient's death was attributable to SBRT toxicity; another patient needed hospital treatment. One patient appeared Grade 5 RILI at 3 months after SBRT and died for respiratory failure (Figure 1). The relationships between the Grade 2 or higher RILI and clinical factors

Table 2 RILI grade in the patients with subclinical ILD, COPD, and PE

\begin{tabular}{|c|c|c|c|c|c|c|}
\hline Patients & \multicolumn{6}{|c|}{ RILI grade ${ }^{a}$} \\
\hline All patients $(n=109)(\%)$ & $12(11.0)$ & $49(51.4)$ & $42(38.5)$ & $3(2.8)$ & $2(1.8)$ & $1(0.9)$ \\
\hline With subclinical ILD (n=38) (\%) & $5(13.2)$ & $15(39.5)$ & $14(36.8)$ & $1(2.6)$ & $2(5.3)$ & $1(2.6)$ \\
\hline With COPD (n=25) (\%) & $4(16.0)$ & $11(44.0)$ & $6(24.0)$ & $2(8.0)$ & $1(4.0)$ & $1(4.0)$ \\
\hline
\end{tabular}

a, CTCAE v5.0. RILI, radiation-induced lung injury; ILD, interstitial lung disease; COPD, chronic obstructive pulmonary disease; PE, pulmonary emphysema. 
Table 3 Characteristics of the patients with extensive pneumonitis

\begin{tabular}{lccccccccccc}
\hline Case & Tumor & $\begin{array}{c}\text { Tumor } \\
\text { size }(\mathrm{cm})\end{array}$ & $\begin{array}{c}\text { Subclinical } \\
\text { ILD, score }\end{array}$ & COPD & PE & $\begin{array}{c}\text { V10 } \\
(\%)\end{array}$ & $\begin{array}{c}\text { V20 } \\
(\%)\end{array}$ & $\begin{array}{c}\text { Lung MLD } \\
(\mathrm{Gy})\end{array}$ & $\begin{array}{c}\text { Contralateral } \\
\text { MLD (Gy) }\end{array}$ & $\begin{array}{c}\text { Total dose } \\
\text { (Gy/fraction) }\end{array}$ & $\begin{array}{c}\text { Toxicity } \\
\text { grade }\end{array}$ \\
\hline 1 & Primary & 1.4 & Yes, 1 & No & No & 11.4 & 8.1 & 6.6 & 0.5 & $50 / 10$ & 4 \\
2 & Primary & 4.0 & Yes, 1 & Yes & Yes & 13.7 & 7.4 & 5.6 & 0.9 & $40 / 5$ & 5 \\
3 & Primary & 1.5 & Yes, 1 & Yes & No & 13.1 & 7.2 & 5.5 & 0.6 & $40 / 5$ & 4 \\
\hline
\end{tabular}

RILI, radiation-induced lung injury; ILD, interstitial lung disease; COPD, chronic obstructive pulmonary disease; PE, pulmonary emphysema; MLD, mean lung dose.
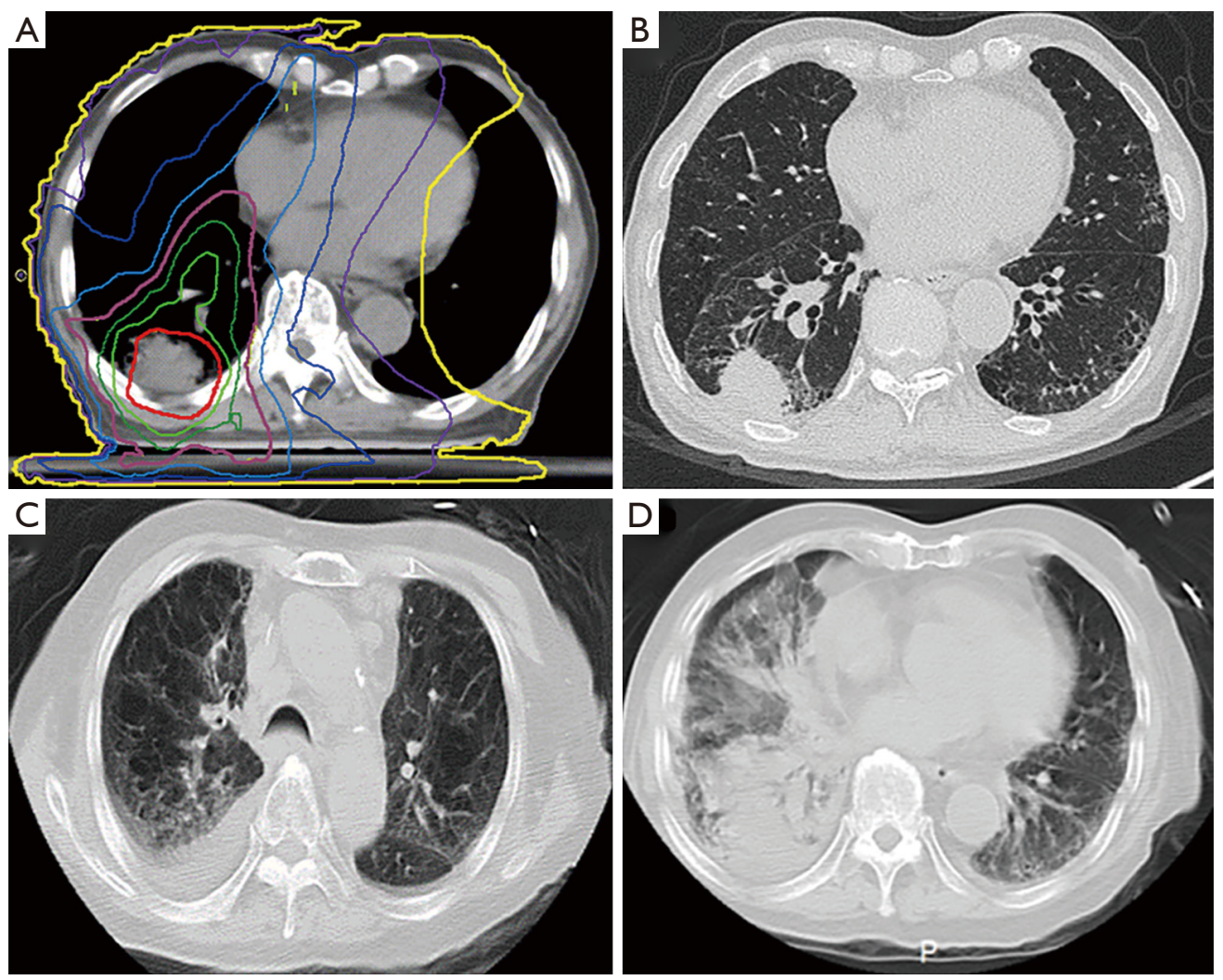

Figure 1 The CT appearance of extensive pneumonitis. (A) Dose distribution on computed tomography (CT). Red and yellow lines are 40 and 2 Gy, respectively. (B) CT image before stereotactic body radiotherapy. CT at the level of bilateral lower lobes shows focal subpleural ground-glass abnormalities. In this case, the abnormality was less than $5 \%$ of the lung (interstitial lung disease score 1); (C,D) 3 months after stereotactic body radiotherapy. CT at the level of the upper lobes (C) and lower lobes (D) showing extensive ground-glass abnormalities and pulmonary fibrosis.

are listed in Table 4. Subclinical ILD was not a significant factor of Grade 2-5 RILI $(\mathrm{P}=0.608)$. In the patients with subclinical ILD, MLD was significantly related to Grade 2 or higher RILI $(\mathrm{P}=0.042)$ (Table S1).

In all patients, the median PFS and OS were 56.3 and 67.9 months, respectively. The PFS and OS rates were $82.5 \%$ and $94.4 \%$ at 1 year, and $65 \%$ and $81.9 \%$ at 3 years, respectively. There was no significant difference in the PFS rate between the patients with subclinical ILD and those without. The PFS rates at 3 years in the subclinical ILD patients and those without ILD were $61.6 \%$ and $66.8 \%$, respectively $(\mathrm{P}=0.266)$ (Figure $2 A)$. No significant difference was found in the PFS among the patients with Grade 0-1 RILI and those with Grade 2-5 RILI ( $\mathrm{P}=0.943)$ (Figure 2B). 
Table 4 Clinical factors associated with RILI

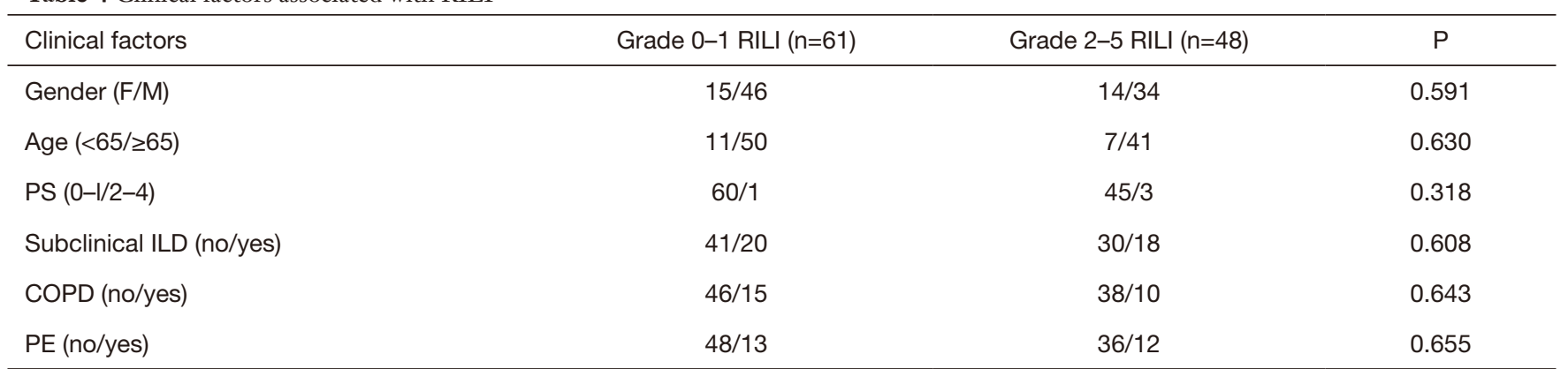

RILI, radiation-induced lung injury; PS, performance status; ILD, interstitial lung disease; COPD, chronic obstructive pulmonary disease; PE, pulmonary emphysema.
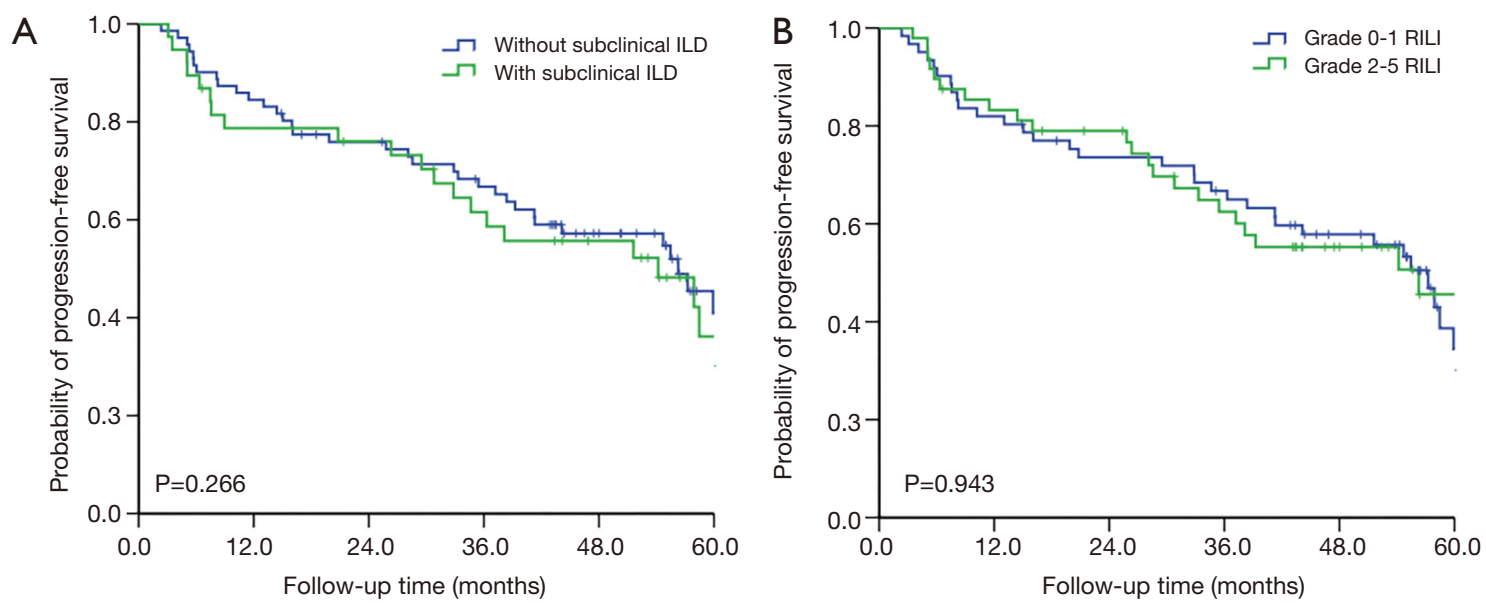

Figure 2 Estimated cumulative incidence curves illustrating (A) progression-free survival (PFS) in the patients with subclinical interstitial lung disease (ILD) patients and those without ILD and (B) PFS in the patients with Grade 0-1 radiation-induced lung injury (RILI) and those with Grade 2-5 RILI by Kaplan-Meier analysis.

\section{Discussion}

Although the toxicity of SBRT is generally low, the number of studies focusing on serious lung toxicities in patients with pre-existing ILD are increasing. These studies are mostly retrospective in nature, heterogeneous in the radiation doses employed, and have an extremely wide range of the treatment-related mortality. Therefore, the risk of SBRT in this setting may be greater than that of standard lung SBRT cases, but the real risk is unclear (14).

Although radiation pneumonia is the main complication after radiotherapy, the incidence of severe radiation pneumonia after SBRT is very few. For patients with coexisting ILD treated with SBRT, Chen et al. reported in a systematic review that the weighted proportions for ILDspecific toxicity and treatment-related mortality were $25 \%$ and $15.5 \%$, respectively (15). Therefore, a growing number of studies have identified co-existing ILD as a predictor of increased treatment-related toxicity.

Glick et al. reported that RILI was significantly higher in patients with co-existing ILD than those patients without ILD (Grade $\geq 2,20.5 \%$ vs. $5.8 \%$; $\mathrm{P}<0.01$; Grade $\geq 3,10.3 \%$ vs. $1.0 \% ; \mathrm{P}<0.01)$. Two of three patients with Grade 5 RILI presented with imaging features of ILD (16). There was a significant relationship between the presence of RILI and subclinical ILD. Okubo et al. found subclinical ILD to be the only significant factor for grade 2-5 RILI, and patients with ILD should be monitored carefully for severe RILI after SBRT treatment (17). In our study, there was no significant relationship between Grade 2-5 RILI and subclinical ILD. However, the patients with subclinical ILD were more likely to appear extensive RILI, even if the subclinical ILD on CT imaging was slight and the dose of 
SBRT was decreased (Table 3). Therefore, for the patients with subclinical ILD receiving SBRT, we should consider the risks of extensive RILI and given sufficient informed consent to these patients for the risks of extensive RILI. After SBRT, RILI must be carefully observed and managed.

Compared with classical RILI, extensive RILI seems to be a completely different disease process, involving genetic factors and immune regulation, while classical RILI is characterized by the inflammatory consequences of direct irradiation injury to lung tissue (18). Roberts et al. showed that lymphocytic alveolitis appeared in both lung fields after strict unilateral thoracic radiotherapy, especially in patients with clinical pneumonia. They drew the conclusion that radiotherapy might cause generalized lymphocyte-mediated hypersensitivity reactions (19). In the current study, though the mean lung dose of the contralateral lung were 0.3-7.1 Gy, however, in the three patients with extensive RILI , they were low $(0.5,0.6$ and 0.9 Gy, respectively), which could be explained by the above non-classical disease process (Table 3).

Nintedanib has been reported to decrease the incidence of acute exacerbations of IPF (20), and has been recommended for the treatment of IPF by China's 2019 "Diagnostic and Treatment Guidelines for Rare Diseases" (21). However, there is no record in the existing literatures of similar studies being performed of the peri-radiotherapy period in the lung cancer patients. Although corticosteroids are a common choice for the treatment of radiation pneumonitis, there is still a lack of evidence from relevant controlled trials supporting its use for the precaution of acute exacerbation of IPF (22).

Dosimetric factors of lung play important roles in determining the risk for symptomatic RILI for patients undergoing SBRT. Several studies had reported MLD was a significant predictor of symptomatic RILI, with values ranging from 4 to 9.14 Gy (23-26). Matsuo et al. reported that the incidence of symptomatic RILI after SBRT treatment was $15.0 \%$ in patients with lung V20 $<5.8 \%$, and $42.9 \%$ in the remaining patients (27). A recent metaanalysis showed that in early stage lung cancer patients with co-existing ILD treated with SBRT, V $20<6.5 \%$ and MLD $<4.5$ Gy were found to be metrics associated with reduced mortality and treatment-related ILD-specific toxicity (15). In the current study, dosimetric factor, such as MLD, was also significantly related to RILI in patients with subclinical ILD (Table S1). All three patients with Grade 4-5 extensive RILI exhibited high lung V10 (11.4\%, 13.7\%, and 13.1\%),
V20 (8.1\%, 7.4\%, and 7.2\%), and MLD (6.6, 5.6, and $5.5 \mathrm{~Gy}$ ) values (Table 3). Therefore, we suggest that safer values of dosimetric factors should be used in patients with subclinical ILD treated with SBRT to prevent these patients from developing extensive RILI.

The limitations of this study are as follows. First, this was a small-sample retrospective study, and the possibility of selection bias regarding the predictive factors cannot be ignored. Secondly, because the data of patients with active ILD were not properly recorded, we could not analyze their data separately from SBRT. Furthermore, these results should be explained in conjunction with the natural incidence of acute exacerbations among the general IPF population, which has been estimated to be in the annual range of $5 \%$ to $19 \%$ (28). Finally, we have completed a further in-depth analysis of the individualized clinical conditions of patients. It is warrant to realize the possibility of an increased SBRT-related death risk in different cohorts with subclinical ILD. It is necessary to conduct further large-sample prospective research to clarify this concern. Therefore, in order to evaluate the therapeutic efficacy of SBRT in T1-2N0M0 NSCLC patients with ILD who are not suitable for surgical treatment, we have conducted prospective clinical cohort study. The novelty of this study is that it used the ILD-GAP score to stratify patients, thereby incorporating the risk of death from ILD based on age, physiology, and gender, and combined with the patient's peripheral blood biomarkers and radiotherapy physical dosimetry parameters to establish a precise and individualized dose-survival prediction model (29).

In summary, we found that subclinical ILD was not a predictor of Grade 2-5 RILI in patients received SBRT; however, patients with subclinical ILD may develop uncommon extensive and fatal RILI beyond the irradiated field. No significant differences in the PFS rates were presented between patients with subclinical ILD and patients without subclinical ILD. Therefore, formal studies are needed to determine the efficacy, prognostic factors, and toxicity of SBRT in patients with subclinical ILD.

\section{Acknowledgments}

Funding: This work was supported by the Start-up Fund for Talent Introduction of Shanghai Pulmonary Hospital (YX) (grant number: 201801) and Science Research Foundation of China Ministry of Health - Zhejiang Medicine \& Health Key Research Fund (No. 201339868). 


\section{Footnote}

Reporting Checklist: The authors have completed the STROBE reporting checklist. Available at http://dx.doi. org/10.21037/tlcr-20-1050

Data Sharing Statement: Available at http://dx.doi. org/10.21037/tlcr-20-1050

Conflicts of Interest: All authors have completed the ICMJE uniform disclosure form (available at http://dx.doi. org/10.21037/tlcr-20-1050). The authors have no conflicts of interest to declare.

Ethical Statement: The authors are accountable for all aspects of the work in ensuring that questions related to the accuracy or integrity of any part of the work are appropriately investigated and resolved. All procedures performed in this study involving human participants were in accordance with the Declaration of Helsinki (as revised in 2013). The study was approved by institutional/regional/ national ethics/committee/ethics board of Shanghai Pulmonary Hospital (No.: L20-320Y) and individual consent for this retrospective analysis was waived.

Open Access Statement: This is an Open Access article distributed in accordance with the Creative Commons Attribution-NonCommercial-NoDerivs 4.0 International License (CC BY-NC-ND 4.0), which permits the noncommercial replication and distribution of the article with the strict proviso that no changes or edits are made and the original work is properly cited (including links to both the formal publication through the relevant DOI and the license). See: https://creativecommons.org/licenses/by-nc-nd/4.0/.

\section{References}

1. Raghu G, Mageto YN, Lockhart D, et al. The accuracy of the clinical diagnosis of new-onset idiopathic pulmonary fibrosis and other interstitial lung disease: A prospective study. Chest 1999;116:1168-74.

2. Naccache JM, Gibiot Q, Monnet I, et al. Lung cancer and interstitial lung disease: a literature review. J Thorac Dis 2018;10:3829-44.

3. Choi S, Park J. Surgical outcomes and prognosis of nonsmall-cell lung cancer in patients with chronic lung diseases: a retrospective analysis. Eur J Cardiothorac Surg 2020;58:357-64.
4. Fujita T, Kuroki T, Hayama N, et al. Pemetrexed Plus Platinum for Patients With Advanced Non-small Cell Lung Cancer and Interstitial Lung Disease. In Vivo 2019;33:2059-64.

5. Goodman CD, Nijman SFM, Senan S, et al. A Primer on Interstitial Lung Disease and Thoracic Radiation. J Thorac Oncol 2020;15:902-13.

6. Kolek V, Vašáková M, Šterclová $M$, et al. Radiotherapy of Lung Tumours in Idiopathic Pulmonary Fibrosis. Klin Onkol 2017;30:303-6.

7. Okami J, Shintani Y, Okumura M, et al. Demographics, Safety and Quality, and Prognostic Information in Both the Seventh and Eighth Editions of the TNM Classification in 18,973 Surgical Cases of the Japanese Joint Committee of Lung Cancer Registry Database in 2010. J Thorac Oncol 2019;14:212-22.

8. Hanibuchi M, Yamaguchi T, Okada T, et al. Clinical Examination of Acute Exacerbation of Idiopathic Interstitial Pneumonia (IIP) Combined With Lung Cancer After Anti-cancer Treatment. Haigan 2001;41:281-6.

9. Ball D, Mai GT, Vinod S, et al. Stereotactic ablative radiotherapy versus standard radiotherapy in stage 1 nonsmall-cell lung cancer (TROG 09.02 CHISEL): a phase 3 , open-label, randomised controlled trial. Lancet Oncol 2019;20:494-503.

10. Yamaguchi S, Ohguri T, Ide S, et al. Stereotactic body radiotherapy for lung tumors in patients with subclinical interstitial lung disease: the potential risk of extensive radiation pneumonitis. Lung Cancer 2013;82:260-5.

11. National Cancer Institute (U.S.). Common Terminology Criteria for Adverse Events (CTCAE), version 5.0. Bethesda, Md.: U.S. Dept. of Health and Human Services, National Institutes of Health, National Cancer Institute, 2017.

12. Dahele M, Palma D, Lagerwaard F, et al. Radiological changes after stereotactic radiotherapy for stage I lung cancer. J Thorac Oncol 2011;6:1221-8.

13. Ronden MI, Palma D, Slotman BJ, et al. Brief Report on Radiological Changes following Stereotactic Ablative Radiotherapy (SABR) for Early-Stage Lung Tumors: A Pictorial Essay. J Thorac Oncol 2018;13:855-62.

14. Thompson M, Rosenzweig KE. The evolving toxicity profile of SBRT for lung cancer. Transl Lung Cancer Res 2019;8:48-57.

15. Chen H, Senan S, Nossent EJ, et al. Treatment-Related Toxicity in Patients With Early-Stage Non-Small Cell Lung Cancer and Coexisting Interstitial Lung Disease: A Systematic Review. Int J Radiat Oncol Biol Phys 
2017;98:622-31.

16. Glick D, Lyen S, Kandel S, et al. Impact of Pretreatment Interstitial Lung Disease on Radiation Pneumonitis and Survival in Patients Treated With Lung Stereotactic Body Radiation Therapy (SBRT). Clin Lung Cancer 2018;19:e219-e226.

17. Okubo M, Itonaga T, Saito T, et al. Predicting risk factors for radiation pneumonitis after stereotactic body radiation therapy for primary or metastatic lung tumours. Br J Radiol 2017;90:20160508.

18. Morgan GW, Breit SN. Radiation and the lung: a reevaluation of the mechanisms mediating pulmonary injury. Int J Radiat Oncol Biol Phys 1995;31:361-9.

19. Roberts CM, Foulcher E, Zaunders JJ, et al. Radiation pneumonitis: a possible lymphocyte-mediated hypersensitivity reaction. Ann Intern Med 1993;118:696-700.

20. Richeldi L, du Bois RM, Raghu G, et al. Efficacy and safety of nintedanib in idiopathic pulmonary fibrosis. $\mathrm{N}$ Engl J Med 2014;370:2071-82.

21. National Health Commission of the People's Republic of China. Diagnosis and Treatment Guidelines for Rare Diseases. (EB/OL). (2019-02-27). Available online: http:// wwwnhcgovcn/yzygj/s7659/201902/61d06b4916c348e081 0ce1fceb844333shtml

22. Collard HR, Ryerson CJ, Corte TJ, et al. Acute Exacerbation of Idiopathic Pulmonary Fibrosis. An International Working Group Report. Am J Respir Crit Care Med 2016;194:265-75.

23. Ryckman JM, Baine M, Carmicheal J, et al. Correlation of dosimetric factors with the development of symptomatic radiation pneumonitis in stereotactic body radiotherapy.

Cite this article as: Liu Y, Zhu Y, Wu R, Hu M, Zhang L, Lin Q, Weng D, Sun X, Liu Y, Xu Y. Stereotactic body radiotherapy for early stage non-small cell lung cancer in patients with subclinical interstitial lung disease. Transl Lung Cancer Res 2020;9(6):2328-2336. doi: 10.21037/tlcr-20-1050
Radiat Oncol 2020;15:33.

24. Barriger RB, Forquer JA, Brabham JG, et al. A dosevolume analysis of radiation pneumonitis in nonsmall cell lung cancer patients treated with stereotactic body radiation therapy. Int J Radiat Oncol Biol Phys 2012;82:457-62.

25. Chang JY, Li QQ, Xu QY, et al. Stereotactic ablative radiation therapy for centrally located early stage or isolated parenchymal recurrences of non-small cell lung cancer: how to fly in a "no fly zone". Int J Radiat Oncol Biol Phys 2014;88:1120-8.

26. Chang JY, Liu H, Balter P, et al. Clinical outcome and predictors of survival and pneumonitis after stereotactic ablative radiotherapy for stage I non-small cell lung cancer. Radiat Oncol 2012;7:152.

27. Matsuo Y, Shibuya K, Nakamura M, et al. Dose--volume metrics associated with radiation pneumonitis after stereotactic body radiation therapy for lung cancer. Int J Radiat Oncol Biol Phys 2012;83:e545-9.

28. Hyzy R, Huang S, Myers J, et al. Acute exacerbation of idiopathic pulmonary fibrosis. Chest 2007;132:1652-8.

29. Chinese Clinical Trial Registry. A single-center prospective clinical study on the effectiveness and safety of individualized body stereotactic radiotherapy for early stage non-small cell lung cancer with interstitial lung disease. (2020-8-22). Registration number: ChiCTR2000036297. Available online: http://www.chictr. org.cn/showproj.aspx?proj=58733

(English Language Editor: J. Gray) 